Article

\title{
An Internet-Based Survey of Influenza Vaccination Coverage in Healthcare Workers in China, 2018/2019 Season
}

\author{
Haitao Liu ${ }^{1,+}{ }^{,}$, Yayun Tan ${ }^{2,+}$, Muli Zhang ${ }^{3}$, Zhibin Peng ${ }^{3}$, Jiandong Zheng ${ }^{3}$, Ying Qin ${ }^{3}$, \\ Zhiqiang Guo ${ }^{4}$, Junhua Yao ${ }^{4}$, Fen Pang ${ }^{4}$, Teng Ma ${ }^{4}$, Wenjing Duan ${ }^{4}$, Zhongjie $\mathrm{Li}^{3}$, \\ Luzhao Feng ${ }^{3, *}$ and Mo Hao ${ }^{1, *}$ \\ 1 Research Institute of Health Development Strategies \& Collaborative Innovation Center of Social Risks \\ Governance in Health, Fudan University, Shanghai 200032, China; liuht@nhc.gov.cn \\ 2 Suzhou Center for Disease Control and Prevention, Suzhou 215000, China; tanyayun163@163.com \\ 3 Key Laboratory of Surveillance and Early-Warning on Infectious Disease, Division of Infectious Disease, \\ Chinese Center for Disease Control and Prevention, Beijing 102206, China; zhangmuli163@163.com (M.Z.); \\ pengzb@chinacdc.cn (Z.P.); zhengjd@chinacdc.cn (J.Z.); qinying@chinacdc.cn (Y.Q.); lizj@chinacdc.cn (Z.L.) \\ 4 Beijing Dingxiangyuan Tiantian Health Technology, Beijing 100020, China; guozhiqiang@dxy.cn (Z.G.); \\ yaojh@dxy.cn (J.Y.); pangf@dxy.cn (F.P.); mateng@dxy.cn (T.M.); duan1105@yeah.net (W.D.) \\ * Correspondence: fenglz@chinacdc.cn (L.F.); haomo03@fudan.edu.cn (M.H.); \\ Tel.: +86-10-58900541 (L.F.); +86-21-33561022 (M.H.) \\ + These authors contributed equally to this work.
}

Received: 25 November 2019; Accepted: 24 December 2019; Published: 26 December 2019

\begin{abstract}
Influenza vaccination coverage was low among healthcare workers (HCWs) in China. In October 2018, the National Health Commission of China began to require all hospitals to provide free influenza vaccination for HCWs to increase vaccine uptake, and no study on vaccine coverage among HCWs at the national level after the announcement of new policy. This evaluation aims to investigate self-reported influenza vaccination coverage among HCWs and factors that may affect vaccine receipt during the 2018/2019 influenza season. We delivered an opt-in internet panel survey among registered HCWs of DXY forum (the biggest online forum for HCWs in China). The survey was self-administered using a standard questionnaire to collect information on demographics, occupational characteristics, policy implementation, influenza vaccination and influence factors. We conducted multivariate logistic regression analysis to assess factors associated with receipt of influenza vaccine. The response rate of this online survey was $3.6 \%$. The seasonal influenza vaccine coverage reported among HCWs surveyed during the 2018/2019 season was 11.6\% (472/4078). Only $19.0 \%$ (774/4078) of HCWs surveyed reported free policy in their workplace. Combing free policy and workplace requirement proved to be effective to improve influenza vaccination coverage in HCWs (PR $=6.90,95 \%$ CI: 6.03-7.65). The influenza vaccination coverage among surveyed HCWs in China was low during the 2018/2019 season. To increase future vaccination uptake, we recommend a multi-faceted strategy that include free policy, workplace requirement and promotion, on-site vaccination, and monitoring.
\end{abstract}

Keywords: influenza; healthcare workers; vaccination coverage; internet-based survey; China

\section{Introduction}

Seasonal influenza is estimated to cause 88,100 respiratory deaths annually in China [1]. Although influenza vaccination is the most effective way to prevent influenza infection [2], vaccination coverage in China was estimated to be $1.5-2.2 \%$ among the general population during 2004 and 2014 [3]. 
Healthcare workers (HCWs) are one of the recommended groups for influenza vaccination because of their risk of getting infected and vaccination of HCWs significantly reduced influenza-like illness and all-cause mortality among patients, as well as number of working days saved [4,5]. Regional estimates of influenza vaccination coverage among Chinese HCWs have ranged from 5-18\% [6,7], but data describe national coverage estimates was lacked.

Influenza vaccine is generally paid for out-of-pocket in China and there is no immunization program for influenza nationally. In October 2018, China's National Health Commission issued an official document, requiring all hospitals to provide free influenza vaccination for HCWs [8]. However, the status of the implementation of this policy and its effect on influenza vaccination coverage is unknown. We aimed to investigate the 2018/2019 influenza vaccination coverage, factors associated with influenza vaccination, including workplace vaccination policies, and reasons for influenza vaccine receipt or non-receipt among HCWs.

\section{Materials and Methods}

\subsection{Study Design}

We conducted an opt-in internet based cross-sectional study from 21 March to 15 April 2019, and enrolled HCWs registered on DXY forum. DXY is an online forum for HCWs, medical researchers, teachers, and students. There were approximately 2.3 million registered HCWs, accounting for about $20 \%$ of all the HCWs in mainland China [9]. Our eligibility criteria for participation in the survey required that users list an occupation as doctor, nurse, radiologist, pharmacist, medical technician, administrative support staff, and confirmed their workplace as a hospital. We randomly sampled a proportion of those eligible for participation, and sent them an email invitation letter that included a link to a standardized questionnaire for completion.

We used a simple random sample formula to calculate sample size. Assuming a predicted influenza vaccine coverage among HCWs of $10 \%$ with an allowable error of $1 \%$, we calculated a sample size of 3445 . To allow for disqualification of incomplete questionnaires, we increased the sample size by $10 \%$, with a final target sample population of 3789 .

\subsection{Data Collection}

Participants were required to complete a standardized online questionnaire. Data were collected on healthcare workers demographic and occupational characteristics, self-reported influenza vaccination status during the 2018/2019 influenza season, workplace policies related to influenza vaccination, and reasons for receipt or non-receipt. Respondents were able to select more than one reason and no rank order was required.

Based on the National Influenza Prevention and Control Scheme (pilot version) from China's National Health Commission, we classified respondents who worked in respiratory disease, infectious disease, pediatrics, gynecology, and obstetrics and emergency departments as working in a "high risk department" [8].

For workplace policies related to influenza vaccination, we used the following definitions: (1) Free vaccination was defined as the hospitals or local government paying the vaccine and related service costs. (2) Promotion of vaccination was defined as an employer recommending vaccination through a health communication campaign at a facility level, and (3) requirement for vaccination was defined as a workplace issuing official documents requiring vaccination of HCWs.

For the purposes of comparing our study population to that of the general population of HCWs in China, demographic data on the population of HCWs in China were acquired from the National Health Commission of China in 2018 [10]. Gross domestic product (GDP) per capita of each province was obtained from National Bureau of Statistics in 2018 [11]. 


\subsection{Statistical Analysis}

Influenza vaccination coverage was calculated by dividing the total number of HCWs completing the questionnaires by the number of HCWs reporting vaccine receipt among them. To compare demographic and occupational characteristics, we used Chi-square tests or Fisher exact tests for the categorical variables. Two-sided $p$-value of $<0.05$ was considered statistically significant. Multivariate logistic regression analysis were used to assess factors associated with influenza vaccination. Demographic and occupational characteristics, workplace vaccination policies were included in the regression model as independent variables.

As the prevalence of vaccination was expected to be higher than $10 \%$, the odds ratio (OR) might overestimate the effects of promotion factors compared to prevalence ratios (PR). We calculated PR based on OR using function: $\mathrm{PR}=\frac{\mathrm{OR}}{\left(1-\mathrm{P}_{0}\right)+\left(\mathrm{P}_{0} \times \mathrm{OR}\right)}$ to avoid overestimation [12]. In this function, $\mathrm{P}_{0}$ means the prevalence of vaccination of HCWs without exposure.

Additionally, a new variable was created combing free policy and requirement/promotion. This new variable was a categorical variable with six classes, namely free policy with requirement/promotion/none, no free policy with requirement/promotion/none. Also, multivariate logistic regression analysis were used to assess this new factor with all the other variables as covariables and PR was calculated.

\subsection{Human Subjects Review}

The study protocol and questionnaire were approved by the ethical review committee at the Chinese Center for Disease Control and Prevention (No. 201901, China CDC, Beijing, China).

\section{Results}

\subsection{Demographics of Study Population}

In total, we sent 116,372 invitations to HCWs, of those, 4,370 opened the survey and 4161 responded. The response rate was $3.6 \%(4161 / 116,372)$. Of the HCWs responded, 4078 had completed responses to all questions in the questionnaire, and we used this number as our study population.

The HCWs surveyed covered 2734 hospitals and 31 provinces in mainland China. Of the 4078 HCWs surveyed, 3316 were physicians, 540 were nurses, 178 were HCWs of other clinical professions (mainly radiologists) and 44 were nonclinical personnel. Age, years of work, and job title of the surveyed HCWs were summarized in Table 1, compared with the whole population of HCWs in China.

Table 1. Characteristics of healthcare workers surveyed and in China Health Statistics Yearbook 2018.

\begin{tabular}{ccccc}
\hline Characteristic & Category & $\begin{array}{c}\text { HCWs in } \\
\text { China (\%) }\end{array}$ & $\begin{array}{c}\text { HCWs in This } \\
\text { Study (\%) }\end{array}$ & $\begin{array}{c}p \text {-Value for } \\
\text { Chi-Square Test }\end{array}$ \\
\hline \multirow{5}{*}{ Age in years } & $<25$ & 7.9 & 1.9 & 0.044 \\
& $25-34$ & 38.1 & 34.9 & \\
& $35-44$ & 26.1 & 41.9 & \\
& $45-54$ & 18.6 & 18.3 & \\
Years of working & $55-59$ & 3.8 & 2.1 & \\
& $\geq 60$ & 5.4 & 0.8 & \\
& $<5$ & 22.7 & 12.2 & \\
& $5-9$ & 23.0 & 22.7 & \\
& $10-19$ & 21.3 & 36.0 & \\
& $20-29$ & 19.2 & 23.5 & \\
Job title & $\geq 30$ & 13.7 & 5.7 & \\
& Senior & 1.8 & 6.4 & \\
& Associate-senior & 6.0 & 23.3 & \\
& Middle & 19.6 & 42.0 & \\
& Primary & 29.3 & 24.9 & \\
& Unclassified/unknown & 43.2 & 3.5 & \\
\hline
\end{tabular}


The median age of HCWs surveyed was 37 years (ranged from 18 to 79), the median duration of working was 12 years (ranged from less than 1 to 54). In comparison with the population of HCWs in China, HCWs in this study were older, had longer years of work and higher job title (Table 1).

\subsection{Implementation of Free Vaccination Policy}

Among 4078 respondents, 19.0\% (774/4078) reported hospital offered free vaccination, $35.4 \%$ (1445/4078) reported hospitals promoted influenza vaccination, and 5.4\% (221/4078) reported hospitals required HCWs for vaccination.

\subsection{Influenza Vaccination Coverage among HCWs and Associated Factors}

Among the 4078 HCWs, 472 reported they had received influenza vaccine since September 2018. The overall influenza vaccination coverage among HCWs responding to the survey for 2018/2019 season was $11.6 \%$. HCWs provided with free vaccination policy (32.3\%) had higher vaccine coverage compared with no free policy $(6.7 \%)(p<0.001)$. HCWs with workplaces' requirement $(37.6 \%)$ or promotion (16.8\%) for vaccination had higher vaccine coverage compared with HCWs without workplaces' requirement or promotion $(6.1 \%)(p<0.001)$. HCWs working in "high risk departments" had higher vaccine coverage $(16.3 \%)$ than those not working in high risk departments $(10.2 \%)(p<0.001)$. Among three levels of hospitals, vaccine coverage was highest among primary care $(15.1 \%)$, followed by tertiary care $(11.5 \%)$ and secondary care $(10.3 \%)(p=0.032)$. HCWs working $\geq 3$ years $(11.8 \%)$ were more likely to be vaccinated than those working $<3$ years $(5.8 \%)(p=0.014)$. In addition, HCWs with senior title $(12.2 \%)$ and middle title $(12.7 \%)$ had significantly higher vaccine coverage compared with those with primary and lower title $(9.2 \%)(p=0.013)$ (Table 2).

Multivariate logistic regression analysis was used to assess factors associated with influenza vaccination and prevalence ratio (PR) was calculated to avoid overestimation of promotion effect using odds ratio (OR). Those who were provided free vaccination by their workplace (PR $=3.67,95 \%$ CI: 3.07-4.35), required ( $\mathrm{PR}=3.21,95 \% \mathrm{CI}: 2.48-4.04$ ) or promoted ( $\mathrm{PR}=1.75,95 \%$ CI: $1.43-2.14$ ) by workplace to receive vaccination, working $\geq 3$ years ( $\mathrm{PR}=2.09,95 \% \mathrm{CI}: 1.11-4.03$ ), and working in "higher risk departments" ( $\mathrm{PR}=1.59,95 \% \mathrm{CI}$ : 1.30-1.92) were more likely to get vaccinated (Table 2).

Table 2. Percentage of healthcare workers who received influenza vaccination- Internet panel survey, China, 2018-19 season.

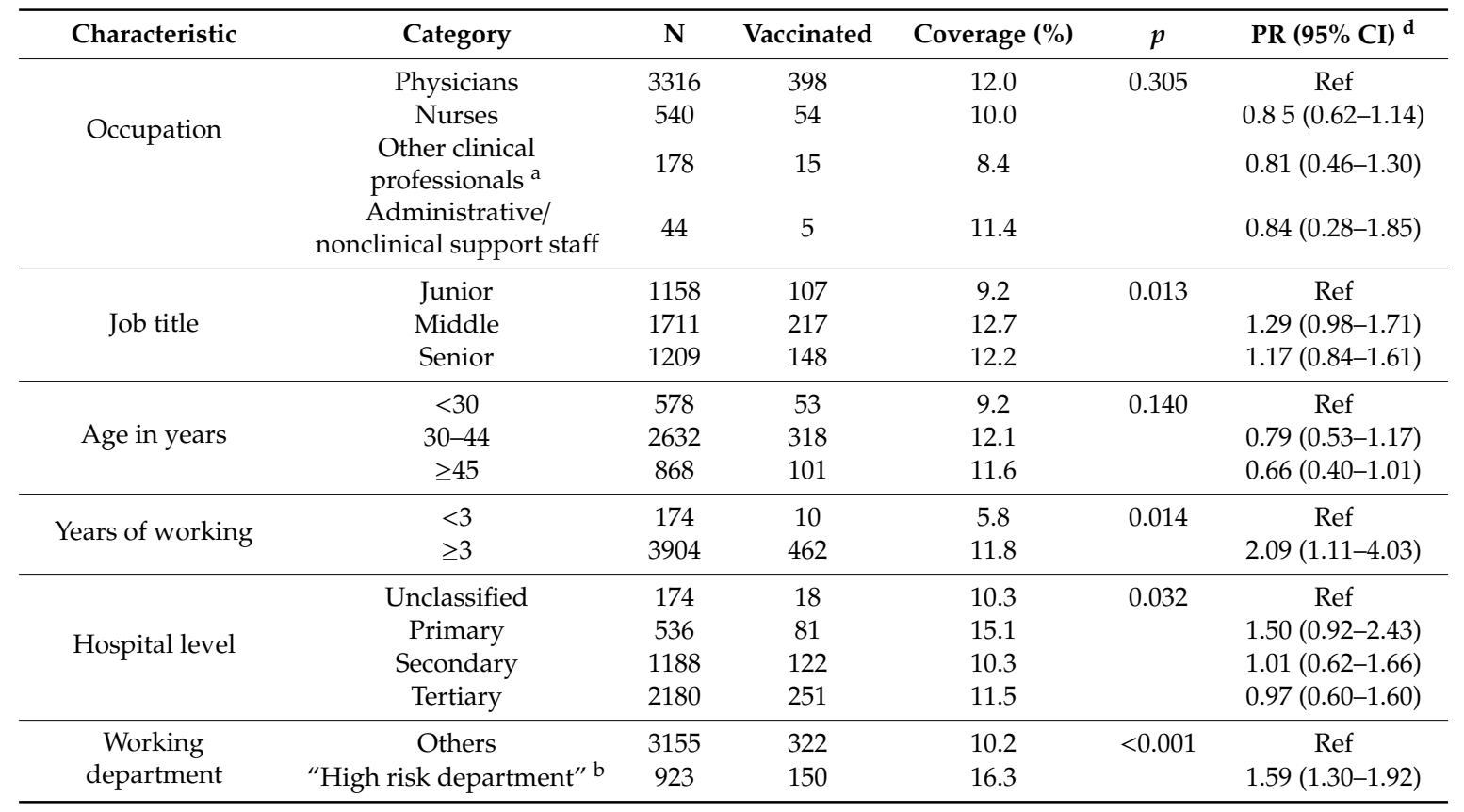


Table 2. Cont.

\begin{tabular}{|c|c|c|c|c|c|c|}
\hline Characteristic & Category & $\mathbf{N}$ & Vaccinated & Coverage $(\%)$ & $p$ & $\operatorname{PR}(95 \% \mathrm{CI})^{d}$ \\
\hline GDP per capita ${ }^{c}$ & Middle & 1193 & 122 & 10.2 & 0.049 & $0.91(0.68-1.21)$ \\
\hline \multirow{2}{*}{$\begin{array}{l}\text { Hospital's } \\
\text { intervention } \\
\text { approach }\end{array}$} & None & 2412 & 147 & 6.1 & \multirow[t]{2}{*}{$<0.001$} & Ref \\
\hline & Promotion & 1445 & 242 & 16.8 & & $1.75(1.43-2.14)$ \\
\hline \multirow{2}{*}{$\begin{array}{l}\text { Hospital providing } \\
\text { free vaccine }\end{array}$} & No & 3304 & 222 & 6.7 & \multirow{2}{*}{$<0.001$} & Ref \\
\hline & Yes & 774 & 250 & 32.3 & & $3.67(3.07-4.35)$ \\
\hline
\end{tabular}

a Other clinical professionals include: Radiologist, pharmacist, and medical technician. ${ }^{b}$ "More risky departments" include respiration, infectious diseases, emergency, pediatrics, gynecology and obstetrics and geriatrics, according to our definition in the method part. ${ }^{\mathrm{c}}$ The 31 provinces of mainland China were divided into 3 categories according to GDP per capita. "High" for Beijing, Shanghai, Tianjin, Jiangsu, Zhejiang, Fujian, Guangdong, Shandong, Inner Mongolia and Chongqing; "Middle" for Hubei, Shaanxi, Jilin, Liaoning, Ningxia, Hunan, Hainan, Hebei, Henan, Jiangxi and Xinjiang; "Low" for Sichuan, Qinghai, Anhui, Heilongjiang, Guangxi, Shanxi, Xizang, Guizhou, Yunnan and Gansu. ${ }^{d}$ Variables included in the multivariable logistic regression include: occupation, job title, age, years of working, hospital level, working department, GDP per capita, hospital's intervention approach, and hospital providing free vaccine.

\subsection{The Impact of Free Vaccination and Workplace's Vaccination Requirement/Promotion on Vaccine Coverage Rate}

The approach of combining free vaccination and workplace's requirement seemed to be the most effective to improve vaccination rate among HCWs compared with other combinations of these two factors (Table 3). When there was free policy, the vaccination coverage of HCWs was $51.9 \%$ with requirement, $32.6 \%$ with promotion, and $16.3 \%$ without requirement or promotion. When there was no free policy, the vaccination coverage was $15.1 \%$ with requirement, $9.2 \%$ with promotion, and $5.3 \%$ without requirement or promotion.

Multivariate logistic regression analysis also suggested that combing free policy and requirement was the most effective for improving influenza vaccination coverage among HCWs. The prevalence ratio for free policy with requirement was 6.90 (95\% CI: 6.03-7.65), free policy with promotion 5.06 (95\% CI: 4.30-5.84), free policy without requirement or promotion 2.80 (95\% CI: 1.99-3.70), no free policy with requirement 2.48 (95\% CI: 1.45-3.68), and no free policy with promotion 1.61 (95\% CI: 1.27-2.02) compared with no free policy without requirement or promotion.

Table 3. Influenza vaccine coverage in healthcare workers by policy options in 2018-2019 season.

\begin{tabular}{cccccc}
\hline Free Vaccination & Workplace's Policy & $\mathbf{N}$ & Vaccinated & Coverage (\%) & PR (95\% CI) ${ }^{\mathbf{a}}$ \\
\hline \multirow{2}{*}{ Yes } & Requirement & 135 & 70 & 51.9 & $6.90(6.03-7.65)$ \\
& Promotion & 467 & 152 & 32.6 & $5.06(4.30-5.84)$ \\
& None & 172 & 28 & 16.3 & $2.80(1.99-3.70)$ \\
\hline \multirow{2}{*}{ No } & Requirement & 86 & 13 & 15.1 & $2.48(1.45-3.68)$ \\
& Promotion & 978 & 90 & 9.2 & $1.61(1.27-2.02)$ \\
& None & 2240 & 119 & 5.3 & Ref \\
\hline
\end{tabular}

a Variables included in the multivariable logistic regression include: occupation, job title, age, years of working, hospital level, working department, GDP per capita, combination of hospital's intervention approach and hospital providing free vaccine.

\subsection{Reasons for Vaccination Receipt/Non-Receipt in the 2018/2019 Season}

Among the 472 respondents who had received influenza vaccine during the 2018/2019 season, $85.0 \%$ believed that the influenza vaccine offered personal protection, $72.3 \%$ believed that vaccination can protect their family members. Only $25.2 \%$ of HCWs reported that their decision to take vaccine was due to their workplace's requirement and $22.67 \%$ was due to free vaccination. (Table 4 ). 
Table 4. Reasons for influenza vaccination receipt- Internet panel survey, China, 2018-2019 season $(\mathrm{N}=472)$.

\begin{tabular}{ccc}
\hline Reasons for Vaccination $^{\mathbf{a}}$ & $\mathbf{N}$ & $\mathbf{\%}$ \\
\hline Protect myself & 401 & 84.96 \\
Protect my family & 341 & 72.25 \\
Protect patients or persons I cared for & 229 & 48.52 \\
Avoid my work capacity being effected by sickness & 215 & 45.55 \\
Be required by workplace & 119 & 25.21 \\
Have free vaccination offered & 107 & 22.67 \\
\hline
\end{tabular}

a These reasons are not mutually exclusive.

The main reason HCWs reported for not receiving influenza vaccine was that they were too busy to get vaccinated $(51.4 \%)$. Other important reasons for not receiving vaccination included a belief that influenza does not cause severe illness (34.7\%), do not want to pay for vaccination (24.1\%), do not know where to get vaccinated $(22.4 \%)$, believing that the influenza vaccine was not effective $(20.4 \%)$, being worried about the side effects $(20.3 \%)$, and considering that influenza could be easily treated with medicine (12.5\%) (Table 5).

Table 5. Reasons for Influenza vaccination non-receipt-Internet panel survey, China, 2018-2019 season $(\mathrm{N}=3606)$.

\begin{tabular}{ccc}
\hline Reasons for Not Getting Vaccinated $^{\text {a }}$ & $\mathbf{N}$ & $\mathbf{\%}$ \\
\hline I am too busy to get vaccinated & 1853 & 51.39 \\
Influenza does not cause severe illness & 1251 & 34.69 \\
Do not want to pay for vaccination & 868 & 24.07 \\
I do not know where I can get vaccinated & 806 & 22.35 \\
The protecting effect of influenza vaccine is not good & 736 & 20.41 \\
I am worried about the side effects & 733 & 20.33 \\
Influenza can be easily treated with medicine & 451 & 12.51 \\
\hline \multicolumn{2}{c}{ a These reasons are not mutually exclusive. }
\end{tabular}

\section{Discussion}

The reported influenza vaccination coverage among surveyed HCWs for 2018/2019 season was $11.6 \%$ according to this internet-based survey. Only 19.0\% HCWs reported their workplace offered free vaccination. In addition, our study reported the free vaccination policy, workplace requirement or promotion, working in "high risk departments" and working $\geq 3$ years were associated with higher vaccination coverage among HCWs. The factor of combing free policy and workplace requirement had the greatest effect on increasing vaccine coverage ( $P R=6.90,95 \% \mathrm{CI}: 6.03-7.65)$. The main barriers to vaccination were too busy to get vaccinated (51.4\%), a belief that influenza did not cause severe illness (34.7\%) and do not want to pay for vaccination (24.1\%).

Vaccination coverage was $11.6 \%$ for HCWs surveyed, this vaccination rate was similar with previous studies conducted in some cities of China [13]. HCWs are one of the recommended groups for influenza vaccine by WHO and China CDC. In October 2018, China's National Health Commission issued an official document, requiring all hospitals to provide free influenza vaccination for HCWs. The vaccination coverage was much lower than that in the United States (78.4\%, 2017-2018) [14]. This difference was probably associated with different vaccination policies in China and the US. Influenza vaccine is generally paid for out-of-pocket in China and there is no immunization program for influenza nationally. While in the US, a large part of hospitals mandate influenza vaccinations for HCWs (61.4\% in 2017) [15], along with other approaches that include education efforts, easy access to vaccines, vaccine promotion, etc.

This study reported that influenza vaccination uptake of HCWs was significantly associated with being provided with free vaccination ( $P R=3.67,95 \%$ CI: $3.07-4.35)$ by workplace. This result 
was in accordance with previous studies in other countries, irrespective of countries' development status $[16,17]$. In addition, the study reported a highest vaccination rate $(51.9 \%)$ among HCWs who were provided with free vaccination and required for vaccination, however, the rate was suboptimal (15.1\%) if HCWs were required but no free vaccine was provided. Furthermore, 24.1\% HCWs who did not take vaccination reported the reason for non-receipt was they did not want to pay for vaccination. These results supported the approach of providing free vaccination as the core component among all interventions to increase influenza vaccine coverage among HCWs [18]. Providing free vaccination for HCW s was required in the document issued by China's National Health Commission in 2018 for the first time. Before that, only few cities in China developed reimbursement policy for influenza vaccination for children and old people [3]. However, there was no clear guidance on its implementation or enforcement for this free vaccination policy at the healthcare facility level. We found that only $19.0 \%$ (774/4078) HCWs reported that they were provided with free vaccination from workplace. It seems that free vaccination policy was not implemented well in large parts of hospitals in 2018/2019 season. Further evaluations will be needed to monitor the effects of the policy in the future.

Our study reported that influenza vaccination uptake of HCWs was significantly associated with workplace requirement $(P R=3.21,95 \% C I$ : $2.48-4.04)$ or promotion for influenza vaccination (PR $=1.75,95 \%$ CI: 1.43-2.14) by workplace. This result was in accordance with previous studies [19]. As mentioned above, combining workplace requirement and free policy seems to be the most effective method for improving influenza vaccination coverage in HCWs. Free vaccination policy is important, but we can see that when there was just free policy without requirement or promotion by workplace, the vaccination coverage was only $16.3 \%$, much lower that $51.9 \%$ when combing with workplace requirement and $32.6 \%$ with promotion. A multi-faceted strategy that include free policy, workplace requirement and promotion, and monitoring should be considered in the future when made guidance on free policy implementation or enforcement [20].

The main reason HCWs reported for not receiving influenza vaccine was that they were too busy to get vaccinated $(51.39 \%)$, and do not know where to get vaccinated $(22.4 \%)$. This result indicated that providing on-site vaccination for HCWs may help to improve vaccine coverage.

Our study finding supported that misconceptions of influenza and influenza vaccine could act as a barrier to higher coverage of influenza vaccination [21]. Among 3606 unvaccinated HCWs, 34.7\% thought that influenza did not cause severe illness, $20.4 \%$ believed that the influenza vaccine was not effective, and $12.5 \%$ considered that influenza could be easily treated with medicine. This study reminded the importance of strengthening health communication on the risks of influenza infection and benefits of influenza vaccination among HCWs.

This study has several limitations. Firstly, the HCWs surveyed were sampled from DXY forum, which has about 2.3 million registered HCWs, representing $20 \%$ of all the HCWs in China. This population is different from the whole population of HCWs in China, this might result in selection bias. The sample population was older, had longer years of work and higher job title, which might result in a higher evaluation of vaccination coverage according to this study. Secondly, the response rate of this online survey was only $3.6 \%$, the respondents may be different from those who did not respond. There may be response bias due to this low response rate. To encourage participation in future studies, we suggest to take activity of forum users into account when delivering questionnaires, because users with low activity may not see the questionnaire at all. Meanwhile, rewards for participants may be useful, too. Thirdly, we took a brief internet-based questionnaire in this survey, thus the number of questions was limited. We did not collect information about the previous history of influenza, knowledge of influenza and influenza vaccination, perception of vaccine efficacy and side-effects or the history of vaccination so there might be confounding factors for vaccination receipt that were not investigated. 


\section{Conclusions}

The current influenza vaccination coverage among HCWs was low in China. To increase future vaccination uptake, we recommend providing free vaccination for HCWs combined with workplace requirement or promotion, on-site vaccination, improving health communication on the risks of influenza infection and benefits of influenza vaccination among HCWs in China. Further evaluations will be needed to monitor the effects of the policy in the future and should focus on the effect of different interventions such as free policy, on-site vaccination and mandatory vaccination on influenza vaccine receipt among HCWs.

Author Contributions: Conceptualization, H.L., Y.T. and L.F.; methodology, H.L., Y.T., L.F., M.H. and Z.L.; formal analysis, Y.T.; investigation, M.Z., J.Z., Z.G., J.Y., F.P., T.M. and W.D.; data curation, M.Z., J.Z., Z.G., J.Y., F.P., T.M. and W.D.; writing —original draft preparation, H.L. and Y.T.; writing—review and editing, Y.Q., Z.P., L.F. and M.H.; supervision, L.F., M.H. and Z.L.; project administration, Y.Q., Z.P., L.F. and Z.L.; funding acquisition, L.F. and Z.L. All authors have read and agreed to the published version of the manuscript.

Funding: This research was funded by National Science and Technology Major Project of China (2018ZX10713001-005), China Center for Disease Control and Prevention's Key Laboratory of Surveillance and Early-warning on Infectious Disease.

Acknowledgments: Technical support by DXY forum, Alexander J. Millman (Influenza Division, Centers for Disease Control and Prevention, U.S.A.), and Ying Song (Influenza Division, Centers for Disease Control and Prevention, U.S.A.) is gratefully acknowledged.

Conflicts of Interest: The authors declare no conflict of interest. The funders had no role in the design of the study in the collection, analyses, or interpretation of data; in the writing of the manuscript, or in the decision to publish the results.

\section{References}

1. Li, L.; Liu, Y.; Wu, P.; Peng, Z.; Wang, X.; Chen, T.; Wong, J.Y.; Yang, J.; Bond, H.S.; Wang, L.; et al. Influenza-associated excess respiratory mortality in China, 2010-2015: A population-based study. Lancet Public Health 2019, 4, e473-e481. [CrossRef]

2. World Health Organization. Vaccines against influenza WHO position paper-November 2012. Wkly. Epidemiol. Rec. 2012, 87, 461-476.

3. Yang, J.; Atkins, K.E.; Feng, L.; Pang, M.; Zheng, Y.; Liu, X.; Cowling, B.J.; Yu, H. Seasonal influenza vaccination in China: Landscape of diverse regional reimbursement policy, and budget impact analysis. Vaccine 2016, 34, 5724-5735. [CrossRef] [PubMed]

4. Ahmed, F.; Lindley, M.C.; Allred, N.; Weinbaum, C.M.; Grohskopf, L. Effect of influenza vaccination of healthcare personnel on morbidity and mortality among patients: Systematic review and grading of evidence. Clin. Infect. Dis. 2014, 58, 50-57. [CrossRef] [PubMed]

5. Kliner, M.; Keenan, A.; Sinclair, D.; Ghebrehewet, S.; Garner, P. Influenza vaccination for healthcare workers in the UK: Appraisal of systematic reviews and policy options. BMJ Open 2016, 6, e12149. [CrossRef] [PubMed]

6. Lee, P.H.; Cowling, B.J.; Yang, L. Seasonal influenza vaccination among Chinese health care workers. Am. J. Infect. Control 2017, 45, 575-578. [CrossRef] [PubMed]

7. Song, Y.; Zhang, T.; Chen, L.; Yi, B.; Hao, X.; Zhou, S.; Zhang, R.; Greene, C. Increasing seasonal influenza vaccination among high risk groups in China: Do community healthcare workers have a role to play? Vaccine 2017, 35, 4060-4063. [CrossRef] [PubMed]

8. China's National Health Commission. National Influenza Prevention and Control Scheme (Pilot Version). Available online: http://www.nhc.gov.cn/jkj/s7923/201810/b30b71408e5641c7a166d4e389318103.shtml (accessed on 22 November 2019).

9. DXY. About DXY. Available online: http://www.dxy.cn/pages/about.html (accessed on 22 November 2019).

10. China's National Health Commission. China Health Statistics Yearbook 2018; Peking Union Medical College Press: Beijing, China, 2018; pp. 31-32.

11. National Bureau of Statistics of China. National Data. Available online: http://data.stats.gov.cn/easyquery. htm?cn=E0103 (accessed on 22 November 2019). 
12. Zhang, J.; Yu, K.F. What's the relative risk? A method of correcting the odds ratio in cohort studies of common outcomes. JAMA 1998, 280, 1690-1691. [CrossRef] [PubMed]

13. Song, X.; Zhang, L.; Shen, T.; Luo, H. Review of Coverage Rates of Influenza Vaccine among Healthcare Workers and Influencing Factors. Chin. J. Vaccines Immun. 2016, 22, 94-98. (In Chinese)

14. Black, C.L.; Yue, X.; Ball, S.W.; Fink, R.V.; de Perio, M.A.; Laney, A.S.; Williams, W.W.; Graitcer, S.B.; Fiebelkorn, A.P.; Lu, P.J. Influenza Vaccination Coverage Among Health Care Personnel-United States, 2017-2018 Influenza Season. MMWR Morb. Mortal. Wkly. Rep. 2018, 67, 1050-1054. [CrossRef] [PubMed]

15. Greene, M.T.; Fowler, K.E.; Ratz, D.; Krein, S.L.; Bradley, S.F.; Saint, S. Changes in Influenza Vaccination Requirements for Health Care Personnel in US Hospitals. JAMA Netw. Open 2018, 1, e180143. [CrossRef] [PubMed]

16. Palache, A. Seasonal influenza vaccine provision in 157 countries (2004-2009) and the potential influence of national public health policies. Vaccine 2011, 29, 9459-9466. [CrossRef] [PubMed]

17. Fedson, D.S.; Hirota, Y.; Shin, H.K.; Cambillard, P.E.; Kiely, J.; Ambrosch, F.; Hannoun, C.; Leese, J.; Sprenger, M.J.; Hampson, A.W.; et al. Influenza vaccination in 22 developed countries: An update to 1995. Vaccine 1997, 15, 1506-1511. [CrossRef]

18. Hollmeyer, H.; Hayden, F.; Mounts, A.; Buchholz, U. Review: Interventions to increase influenza vaccination among healthcare workers in hospitals. Influenza Other Respir. Viruses 2013, 7, 604-621. [CrossRef] [PubMed]

19. Boey, L.; Bral, C.; Roelants, M.; De Schryver, A.; Godderis, L.; Hoppenbrouwers, K.; Vandermeulen, C. Attitudes, believes, determinants and organisational barriers behind the low seasonal influenza vaccination uptake in healthcare workers-A cross-sectional survey. Vaccine 2018, 36, 3351-3358. [CrossRef] [PubMed]

20. To, K.W.; Lai, A.; Lee, K.C.; Koh, D.; Lee, S.S. Increasing the coverage of influenza vaccination in healthcare workers: Review of challenges and solutions. J. Hosp. Infect. 2016, 94, 133-142. [CrossRef] [PubMed]

21. Martinello, R.A.; Jones, L.; Topal, J.E. Correlation between healthcare workers' knowledge of influenza vaccine and vaccine receipt. Infect. Control Hosp. Epidemiol. 2003, 24, 845-847. [CrossRef]

(C) 2019 by the authors. Licensee MDPI, Basel, Switzerland. This article is an open access article distributed under the terms and conditions of the Creative Commons Attribution (CC BY) license (http://creativecommons.org/licenses/by/4.0/). 